\title{
Evaluation of Clinitek 200 and Rapimat II/T for screening for urinary tract infection
}

\author{
R A Bowman, T V Riley
}

\begin{abstract}
Two machines, the Clinitek 200 and the Rapimat II/T, were evaluated for their ability to screen urine samples for significant bacteriuria and other elements indicative of urinary tract pathology. The automated screening procedures were compared with a conventional approach of microscopy and quantitative culture for 1020 urine specimens obtained from patients in a 700 bed general hospital. When compared with the bacterial culture method both machines gave identical results with a negative predictive value of 0.99 , while when compared with microscopy alone the Clinitek 200 and Rapimat II/T gave negative predictive values of 0.92 and 0.87 , respectively. It is concluded that both machines would provide cost effective screening of urine specimens.
\end{abstract}

Investigation of urinary tract infection remains a major component of the workload in a clinical bacteriology laboratory. Increasing pressures for cost effective diagnostic procedures have precipitated the introduction of numerous screening systems for the early detection of bacteriuria or pyuria or both. ${ }^{1}$ Of these screening methods, the use of multipatch strips has received much attention, particularly strips detecting leucocyte esterase and nitrite. ${ }^{2}$ Recent evaluations of this type of approach have produced a wide range of results. ${ }^{3-6}$ Several factors have probably contributed to this divergence of opinion including the definition of significant bacteriuria and pyuria, different microscopic and culture methods, and different patient populations. Subjective visual assessment of the strip reactions may also have been an important but less measurable factor.

In an effort to reduce subjectivity and individual operator bias in reading test strips the use of automated reading machines has been suggested. ${ }^{67} \mathrm{We}$ report here an evaluation of two of these machines, the Clinitek 200 (Ames Inc, Elkhart, Indiana, USA) and the Rapimat II/T (Behring, Marburg, Germany), as screening procedures for the detection of significant bacteriuria. The systems were also evaluated for their ability to screen urine samples for the presence of other elements indicative of urinary tract pathology.

\section{Methods}

The study population consisted of adult male and female patients attending Sir Charles Gairdner Hospital, a 700 bed general hospital.

A total of 1020 urine specimens were processed during the trial. Most of these were midstream urines; specimens collected from urinary catheters were also included and processed in an identical manner. Specimens were collected into sterile containers and stored at $4^{\circ} \mathrm{C}$ in either the ward or laboratory until processed. All specimens in the study were processed within four hours of collection.

\section{QUANTITATIVE CULTURE}

Urine specimens were cultured by using a $1 \mu \mathrm{l}$ calibrated loop to inoculate a cysteine lactose electrolyte deficient (CLED) (Oxoid Pty Ltd, Basingstoke, Hampshire) agar plate. After incubation at $37^{\circ} \mathrm{C}$ for 24 hours the viable count was determined and significant isolates were identified by established techniques. Growth was regarded as significant if the viable count was more than $10^{5} \mathrm{cfu} / \mathrm{ml}$.

\section{MICROSCOPY}

For microscopical examination, $10 \mathrm{ml}$ of urine was centrifuged at $1500 \mathrm{rpm}(300 \times \mathrm{g})$ for five minutes. The supernatant was decanted and the sediment resuspended in the remaining urine (about $0 \cdot 1 \mathrm{ml}$ ) and examined by phase contrast microscopy. Leucocytes and erythrocytes were recorded as numbers per high power field ( $\times 400$ magnification). More than 5 leucocytes or erythrocytes per high power field was regarded as significant pyuria or haematuria. The presence of casts, other than hyaline, was also considered significant. If pyuria or bacteriuria, or both, were evident, the cover slip was removed, the deposit allowed to air dry and Gram stained. Additional media were inoculated or a direct susceptibility test was performed if indicated.

\section{AUTOMATED SCREENING}

The Clinitek 200 was used to read Multistix 7 (Ames) according to the manufacturer's instructions. Similarly, the Rapimat II/T was used to read Total Screen L strips (Behring).

\section{Results}

SCREENING COMPARED WITH CULTURE

Of 223 specimens positive by culture, 216 were positive for either leucocyte esterase or nitrite using the Clinitek 200. Of the 797 specimens negative by culture, 603 were negative for both leucocyte esterase and nitrite. Performance indices were as follows: sensitivity 0.97 , negative predictive value 0.99 , specificity 0.76 , 
and positive predictive value 0.53 . Using the Rapimat II/T, 213 of 223 specimens positive by culture were positive for either leucocyte esterase or nitrite. Of the 797 specimens negative by culture, 608 were negative for both leucocyte esterase and nitrite. Performance indices were as follows: sensitivity 0.96 , negative predictive value 0.99 , specificity 0.76 , and positive predictive value $0 \cdot 53$. These data are summarised in table 1.

\section{SCREENING COMPARED WITH MICROSCOPY}

Using the Clinitek 200, 476 of 507 specimens with positive microscopy were positive for at least one of leucocyte esterase, nitrite, or blood. Of the 513 specimens with negative microscopy, 336 were negative for all the three variables. Sensitivity was 0.94 , negative predictive value 0.92 , specificity 0.65 , and positive predictive value $0 \cdot 73$. Similarly, with the Rapimat II/T, 451 of the 507 specimens with positive microscopy were positive for at least one of leucocyte esterase, nitrite, or blood. Three hundred and ninety of 513 specimens with negative microscopy were negative for all three variables. Sensitivity was $0 \cdot 89$, negative predictive value 0.87 , specificity 0.75 , and positive predictive value $0 \cdot 77$. These results are summarised in table 2.

\section{Discussion}

The purpose of rapid urine screening is to eliminate negative specimens quickly and

Table 1 Comparison of bacterial culture with Clinitek 200 and Rapimat $I I / T$ for detecting significant bacteriuria

\begin{tabular}{lll}
\hline & \multicolumn{2}{l}{ Culture } \\
\cline { 2 - 3 } & Positive & Negative \\
\hline $\begin{array}{l}\text { Leucocyte esterase } \\
\text { or }\end{array}$ & 216 & 194 \\
$\begin{array}{l}\text { Nitrite positive } \\
\text { Clinitek 200 } \\
\text { Leucocyte esterase } \\
\text { and }\end{array}$ & & \\
$\begin{array}{l}\text { Nitrite negative } \\
\text { Leucocyte esterase } \\
\text { or } \\
\text { Nitrite positive }\end{array}$ & 7 & 603 \\
$\begin{array}{l}\text { Rapimat II/T } \\
\text { Leucocyte esterase } \\
\text { and }\end{array}$ & 213 & 189 \\
Nitrite negative & 10 & 608 \\
\hline
\end{tabular}

Table 2 Comparison of microscopy with Clinitek 200 and Rapimat II/T for detecting abnormalities in urine

\begin{tabular}{ccc}
\hline & \multicolumn{2}{c}{ Microscopy } \\
\cline { 2 - 3 } & Positive $^{\star}$ & Negative \\
\hline $\begin{array}{c}\text { Positive } \dagger \\
\text { Clinitek 200 } \\
\text { Negative }\end{array}$ & 476 & 177 \\
$\begin{array}{c}\text { Positive } \dagger \\
\text { Rapimat II/T } \\
\text { Negative }\end{array}$ & 31 & 336 \\
& 451 & 123 \\
\end{tabular}

*5 leucocytes or erythrocytes/high power field, casts other than hyaline.

†Leucocyte esterase, nitrite, or blood patches positive. cheaply, allowing the laboratory to direct more effort into positive specimens. For the physician, this should mean prompt reporting of normal specimens and improvement in the quality of patient care.

Over the past decade there has been a deluge of reports of rapid urine screening systems. Due to the great variations in patient populations, methods used, and interpretative criteria, opinion is polarised over the possible role these systems should have in the clinical bacteriology laboratory. We therefore chose to evaluate two similar urine screening machines to determine their possible application in our laboratory which provides a service for a medium sized general hospital.

For this study we used $10^{5} \mathrm{cfu} / \mathrm{ml}$ as the breakpoint for significant bacteriuria. ${ }^{8}$ Using $10^{2}$ or $10^{3} \mathrm{cfu} / \mathrm{ml}$ as the breakpoint was not considered because the numbers of young adult women presenting with acute urethral syndrome in our patient population is very low. ${ }^{9}$ Using the criteria outlined above, both systems were effective as screening procedures for detecting significant bacteriuria. Indeed, the machines produced almost identical results. Sensitivity was satisfactory with a negative predictive value of 0.99 in both cases, but specificity was less favourable ( 0.76 for both) with a positive predictive value of 0.53 for both.

The task of performing urinalysis falls to either the bacteriology or chemistry laboratories. In Australia both urine culture and microscopy is normally done in the bacteriology laboratory. For this reason we also evaluated both machines for their ability to detect pathological elements in urine other than bacteria. Again, the machines produced similar results. The Clinitek 200 was slightly more sensitive than the Rapimat II/T, but this was at the expense of specificity.

Both machines had less than optimal negative predictive values (Clinitek 0.92, Rapimat II/T 0.87) and, using the criteria defined initially (more than 5 erythrocytes or leucocytes per high power field, presence of casts other than hyaline), lacked the sensitivity of an effective screening procedure. Closer scrutiny of the false negative results, however, showed that most were marginal, in that both the machines missed mostly urines containing only 5 erythrocytes or leucocytes per high power field. Excluding these marginal results, negative predictive values improved to 0.99 for the Clinitek 200 and 0.97 for the Rapimat II/T, acceptable figures for a screening procedure. The suitability of either machine for the detection of elements other than bacteria therefore depends on the criteria chosen for a positive result. These criteria will also depend on other factors such as the patient population.

In addition to being accurate, a screening method must also be cost effective. We therefore compared the cost of screening urines in the manner we described with the cost of processing the 1020 trial specimens by our conventional method. If all specimens were examined by routine microscopy and culture, we estimated the cost of processing to be $\$ 1800$. If microscopy and culture were performed on 
the $56 \%$ of specimens positive on screening by Rapimat II/T, or the $64 \%$ positive on screening by Clinitek 200, then the cost of processing would have been $\$ 1360$ and $\$ 1555$, respectively, a cost saving of about $20 \%$. Both machines also permit on-line connection to main-frame computer systems, and samples that meet pre-programmed criteria can be automatically reported. While it is difficult to assess objectively the cost benefits of this kind, they seem to be substantial.

The diversity of methods, patient populations, and clinical criteria for infection used to evaluate urine screening procedures have produced variable results. Accordingly, the laboratory is faced with a difficult decision in choosing a system to suit its specific requirements. In our laboratory, which performs both culture and microscopy of urine specimens from patients in a medium sized general hospital, both the Clinitek 200 and the Rapimat II/T provided an effective screening system for detecting significant bacteriuria. Their ability to detect other elements indicative of urinary tract pathology was less satisfactory but may be adequate in certain other hospital and laboratory settings.
We thank Miss Suzan Arrow and Mr John Timmins for their skilful technical assistance, Miss Desley Britten of Behring Diagnostics Australia Pty Ltd for provision of the Rapimat II/T Mr Philip Baxter of Miles Australia Pty Ltd for provision of the Clinitek 200, and Genevieve O'Halloran for her exper secretarial assistance.

1 Pezzlo M. Detection of urinary tract infections by rapid methods. Clin Microbiol Rev 1988;1:268-80.

2 Smith TK, Hudson AJ, Spencer RC. Evaluation of six screening methods for detecting bacteriuria. J Clin Pathol 1988;41:904-9.

3 Pfaller M, Ringenberg B, Rames L, Hegeman J, Koontz F. The usefulness of screening tests for pyuria in combination with culture in the diagnosis of urinary tract infection. Diagn Microbiol Infect Dis 1987;6:207-15.

4 Jones C, MacPherson DW, Stevens DL. Inability of the Chemstrip LN compared with quantitative urine culture to predict significant bacteriuria. J Clin Microbiol to predict signif

5 Bolann BJ, Sandberg S, Digranes A. Implications of probability analysis for interpreting results of leukocyte probability analysis for interpreting results of leukocyte

6 Stevens M. Screening urines for bacteriuria. Med Lab Sci 1989;46:194-206.

7 Ketter D, Braun C, Gallego F, Stirn-Thoma S. Screening urine before microscopy by automated teststrip preselection: clinical evaluation of the improved Rapimat II/T (Behring). Clin Chem 1988;34:1600-2.

$8 \mathrm{Kass} \mathrm{EH}$. Bacteriuria and the diagnosis of infections of the urinary tract. Arch Intern Med 1957;100:709-14.

9 Stamm WE. Protocol for diagnosis of urinary tract infection: reconsidering the criterion for significant bacteriuria. Urology 1988; (Suppl)32:6-12. 